\title{
On the climatological probability of the vertical propagation of stationary planetary waves
}

\author{
Khalil Karami $^{1}$, Peter Braesicke ${ }^{1}$, Miriam Sinnhuber ${ }^{1}$, and Stefan Versick ${ }^{1,2}$ \\ ${ }^{1}$ Institute for Meteorology and Climate Research, Karlsruhe Institute of Technology, Karlsruhe, Germany \\ ${ }^{2}$ Steinbuch Centre for Computing, Karlsruhe Institute of Technology, Karlsruhe, Germany \\ Correspondence to: Khalil Karami (khalil.karami@kit.edu)
}

Received: 9 October 2015 - Published in Atmos. Chem. Phys. Discuss.: 17 November 2015

Revised: 7 June 2016 - Accepted: 12 June 2016 - Published: 12 July 2016

\begin{abstract}
We introduce a diagnostic tool to assess a climatological framework of the optimal propagation conditions for stationary planetary waves. Analyzing 50 winters using NCEP/NCAR (National Center for Environmental Prediction/National Center for Atmospheric Research) reanalysis data we derive probability density functions (PDFs) of positive vertical wave number as a function of zonal and meridional wave numbers. We contrast this quantity with classical climatological means of the vertical wave number. Introducing a membership value function (MVF) based on fuzzy logic, we objectively generate a modified set of PDFs (mPDFs) and demonstrate their superior performance compared to the climatological mean of vertical wave number and the original PDFs. We argue that mPDFs allow an even better understanding of how background conditions impact wave propagation in a climatological sense. As expected, probabilities are decreasing with increasing zonal wave numbers. In addition we discuss the meridional wave number dependency of the PDFs which is usually neglected, highlighting the contribution of meridional wave numbers 2 and 3 in the stratosphere. We also describe how mPDFs change in response to strong vortex regime (SVR) and weak vortex regime (WVR) conditions, with increased probabilities of the wave propagation during WVR than SVR in the stratosphere. We conclude that the mPDFs are a convenient way to summarize climatological information about planetary wave propagation in reanalysis and climate model data.
\end{abstract}

\section{Introduction}

The impact of the background atmospheric state on planetary wave propagation was first investigated by Charney and Drazin (1961) based on linear wave theory. They showed the importance of the background zonal wind for the vertical propagation of large-scale waves from the troposphere into the stratosphere. They found that vertical propagation of stationary planetary waves can only occur when the zonal mean zonal wind is positive. In addition, a strong stratospheric polar night jet of the Southern Hemisphere during winter will block and possibly reflect large-scale waves. This implies that the zonal mean zonal wind should be smaller than a critical value for vertical propagation. This theory also suggests that large-scale waves (zonal wave number $=1,2$, 3) are more likely to propagate upwards because their associated critical wind speeds are higher. Studies by Matsuno (1970), Lin (1982), Huang and Gambo (2002), Limpasuvan and Hartmann (2000), Hu and Tung (2002), and Dickinson (1969) not only confirmed this theory but also stressed the importance of vertical shear of the zonal mean zonal wind as well as the vertical gradient of the buoyancy frequency for vertical propagation of large-scale waves.

Matsuno (1970) introduced the refractive index for stationary planetary waves (or alternatively vertical wave number) as a diagnostic tool for studying the influence of the background zonal flow on planetary wave propagation. According to linear wave theory planetary waves, away from the source regions, tend to propagate toward the region of large positive vertical wave number squared. The existence of Rossby waves is prohibited where the vertical wave number squared is small or negative, which can happen if the zonal mean 

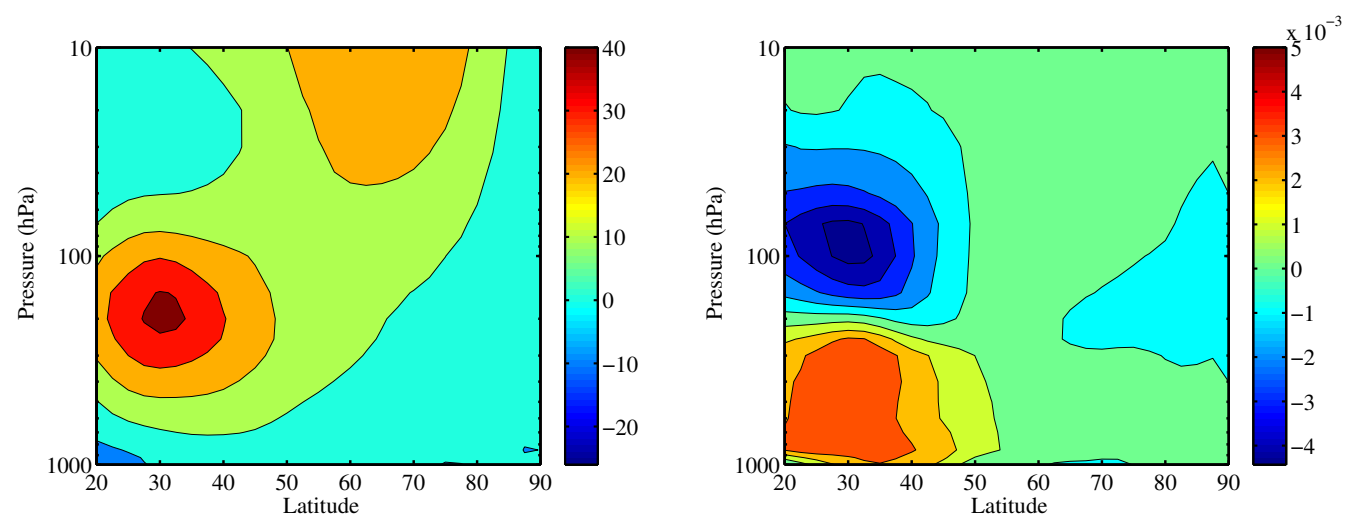

Figure 1. Climatology of the zonal mean zonal wind (left) in and the vertical shear of zonal mean zonal wind (right) for the Northern Hemisphere during DJF. The units are $\mathrm{ms}^{-1}$ for zonal mean zonal wind and $\mathrm{ms}^{-1} \mathrm{~km}^{-1}$ for the vertical shear of zonal mean zonal wind respectively.

zonal wind is easterly, or westerly exceeding the critical wind speed.

The refractive index of Rossby waves as a diagnostic tool provides a framework in which the dynamical forcing of the stratosphere by tropospheric waves can be investigated. However, as shown by Li et al. (2007) the traditional analysis of the refractive index squared makes it difficult, if not impossible, to study the climatological state of the background flow for propagation of planetary waves. In calculating the climatology of the refractive index squared, the problem arises from averaging a time series that could consist of positive and negative values that may cancel each other and hence makes the interpretation of climatologies of this quantity difficult. Another weakness of the vertical wave number is that it is somewhat vague. Randel (1988) pointed out that, while using the vertical wave number as a diagnostic tool one should not overemphasize the details, since it is a qualitative guide. For instance Smith (1983) found that planetary waves can only propagate when and where the vertical wave number squared is positive and very large or avoid the region of large negative values of the vertical wave number. The vagueness arises from vague expressions such as "very large positive" and "very large negative" values of the vertical wave number which demonstrates the arbitrariness of the classic time mean diagnostic.

Here we attempt to address the modeling of such vagueness which has not been previously addressed. We present an algorithm based on fuzzy logic theory which addresses the above-mentioned vagueness and provides an estimate of the favorability of atmospheric background conditions for planetary wave propagation as a function of latitude and altitude. Any diagnostic tool should be consistent with the general knowledge about stationary Rossby wave propagation condition (Table 1). The first and second criterion of Table 1 are the most important findings of the seminal papers of Charney and Drazin (1961) and Matsuno (1970). They made a great contribution on the understanding of the propagation of planetary-scale disturbances from the troposphere into the stratosphere. Eliassen and Palm (1961) based on the wavemean flow interaction theorem showed that the planetary waves also have a strong influence on the zonal mean zonal wind. Matsuno (1970) and Charney and Drazin (1961) argue that only ultra-long waves (wave numbers 1-3) have the capability to propagate from the troposphere into the middle atmosphere. Criterion 3 expresses that the jet maxima block the planetary wave propagation and penetration through the jet maxima is prohibited Karoly and Hoskins (1982). The study of Chen and Robinson (1992) shows that the key parameter that controls the planetary wave propagation is the properties of the tropopause which acts like a valve for the vertical wave propagation from the troposphere into the stratosphere. Furthermore the study of $\mathrm{Hu}$ and Tung (2002) and $\mathrm{Li}$ et al. (2007) indicated that the large positive vertical shear of zonal wind at the tropopause height tends to enhance wave propagation (criterion 4).

Chen and Robinson (1992) and Hu and Tung (2002) have discussed the importance of vertical shear of zonal mean zonal wind on the vertical propagation of Rossby waves. Chen and Robinson (1992) showed that penetration of planetary waves from the troposphere into the stratosphere is sensitive to small changes in the vertical shear of zonal wind near the tropopause height. Hu and Tung (2002) identified that a positive vertical shear of zonal wind enhances wave propagation across the tropopause. Similarly large negative shear of zonal wind tends to trap the planetary waves in the troposphere and hence less is left to penetrate into the stratosphere. Any diagnostic tool that attempts to provide a climatology of stationary Rossby wave propagation conditions should reflect this theory. In fact, we try to develop an algorithm that is capable of demonstrating the enhancing influence of positive vertical shear of zonal wind and impeding influence of negative vertical shear of zonal wind on stationary Rossby wave propagation from the troposphere to the stratosphere. 
Table 1. A summary of known facts about stationary Rossby wave propagation. Any diagnostic tool that attempts to provide a climatology of stationary Rossby wave propagation conditions should be consistent with these criteria. These criteria refer only to the linear waves.

1 For all stationary Rossby waves the most favorable propagation conditions are in the lower troposphere of the mid-latitude region. Upper troposphere and lowermost stratosphere of midlatitude regions are also favorable for Rossby wave propagation.

Matsuno (1970) and Charney and Drazin (1961)

2 For large-scale waves (horizontal and meridional wave numbers 1 to 3 ) the probability to propagate vertically is highest.

3 Rossby waves tend to propagate on the edges of strong westerly Karoly and Hoskins (1982) winds and avoid penetrating through the jet maxima. Therefore, the strong stratospheric polar night jet of the Southern Hemisphere in the winter will block and reflect large-scale waves.

4 Strong vertical shear (positive) is likely to enhance the vertical Chen and Robinson (1992) propagation of waves.

Figure 1 shows the climatology of the zonal mean zonal wind and the vertical shear of zonal mean zonal wind $\left(\mathrm{ms}^{-1} \mathrm{~km}^{-1}\right)$ for the Northern Hemisphere winter months. Northern Hemisphere winter months include December, January and February (DJF) and Southern Hemisphere winter months include June, July and August (JJA). Due to the larger meridional temperature gradient between the tropics and mid-latitudes, the magnitude of the wind shear between 20 and $40^{\circ} \mathrm{N}$ is about four times stronger than the vertical shear at higher latitudes. Regardless of magnitude, it is evident that it is positive in the troposphere and negative in the stratosphere in this latitude band. The importance of the wind shear and buoyancy frequency for the upward wave propagation is discussed by Chen and Robinson (1992).

\section{Data and method}

In the current study we used daily mean zonal wind and temperature from the National Center for Environmental Prediction-National Centre for Atmospheric Research (NCEP-NCAR) (Kalnay et al., 1996) to calculate the vertical wave number of Rossby waves for 50 winters (1961-2010) of both Northern and Southern hemispheres. The vertical wave number for stationary planetary waves is defined as

$$
\begin{aligned}
& m_{k, l}^{2}(y, z)= \\
& \left(\frac{N^{2}}{f^{2} \cos ^{2}(\phi)}\right)\left[\frac{\overline{q_{\phi}}}{\bar{u}}-\left(\frac{k}{a}\right)^{2}-\left(\frac{\pi l}{2 a}\right)^{2}-\left(\frac{f \cos (\phi)}{2 \mathrm{NH}}\right)^{2}\right],
\end{aligned}
$$

where

$$
\begin{aligned}
& \overline{q_{\phi}}= \\
& \cos (\phi)\left[\frac{2 \Omega}{a} \cos (\phi)-\frac{1}{a^{2}} \frac{\partial}{\partial \phi}\left[\frac{\frac{\partial}{\partial \phi}(\bar{u} \cos (\phi))}{\cos (\phi)}\right]\right. \\
& \left.-\frac{f^{2}}{\rho_{0}}\left[\frac{\partial}{\partial z} \frac{\left(\rho_{0} \frac{\partial}{\partial z} \bar{u}\right)}{N^{2}}\right]\right]
\end{aligned}
$$

is the meridional gradient of the zonal mean potential vorticity which is a fundamental quantity in Planetary wave dynamics and the stability of the zonal mean flow (Andrews et al., 1987). Here $H, k, l, \rho_{0}, f, N^{2}, a, \Omega$ and $\phi$ are the scale height, zonal and meridional wave numbers, air density, Coriolis parameter, buoyancy frequency, the Earth's radius and rotation frequency and latitude respectively (Andrews et al., 1987; Matsuno, 1970). The definition of the current version of the vertical wave number of Rossby waves that depends on the two-dimensional wave numbers (zonal and meridional wave numbers) can be found in (Sun et al., 2014; Sun and Li, 2012).

Figures 2 and 3 show the time mean vertical wave number (in the plots weighted with the Earth radius squared) of 50 winters for Northern and Southern hemispheres respectively. The dependence of the time mean vertical wave number on the zonal $(k=1,2,3)$ and meridional wave numbers $(l=1,2,3)$ is visible in both figures. It can be seen that the multi-year average of time mean vertical wave number gives unsatisfactory results. For instance, for $(k, l)=(1,1)$ very high values of the vertical wave number squared are found in high latitudes of the troposphere and the lower stratosphere. Moreover, in most areas of mid- and high latitudes of the troposphere alternating positive and negative values of the vertical wave number squared leads to a noisy structure and makes the interpretation very difficult. The problem originates from overlapping of positive and negative values in the time series and results in a reduction of climatological infor- 
mation. Such features of the time mean vertical wave number are also discussed by others (Mukougawa and Hirooka, 2004; Li et al., 2007). Too high values of time mean vertical wave number northward of $75^{\circ} \mathrm{N}$ in the lower stratosphere are not consistent with criterion 3 in Table 1, because the strong jet is expected to block wave penetration from the troposphere to the stratosphere. The time mean vertical wave number is also not able to capture the meridional wave number dependency on the wave propagation conditions (criterion 2 in Table 1). For example in the Southern Hemisphere, the difference between time mean for wave $(2,1),(2,2)$ and $(2,3)$ in the stratosphere (above $100 \mathrm{hPa}$ ) is small, suggesting no considerable influence from the meridional wave numbers on the vertical propagation of planetary waves from the troposphere to the stratosphere. In the current study, the time mean vertical wave number squared is calculated by the time mean of the instantaneous vertical wave number derived from the daily zonal mean field. As shown in Figs. 2 and 3 the time mean vertical wave number has a noisy structure. One possibility to reduce the noise level is to calculate the vertical wave number of the time-mean zonal mean fields instead (Fig. A3). However time-dependent Rossby waves propagate on the instantaneous atmospheric state and not on the timeaveraged fields. Therefore we focus on an approach to reduce the level of noise in the time-averaged instantaneous vertical wave number.

\section{Probability of positive vertical wave number squared}

Li et al. (2007) introduced the frequency distribution of days with negative vertical wave number squared as an alternative metric to describe how planetary waves can propagate. Figure 4 shows the probabilities of positive vertical wave number squared for Northern Hemisphere winter time expressed as the percentage of days with positive $m_{k, l}^{2}(y, z)$ for wave $(1,1),(1,2)$ and $(1,3)$. By comparing to the time mean of the same waves we conclude that this quantity is capable of describing the required wave properties better than the time mean of $m_{k, l}^{2}(y, z)$. However, it results in high values of probability between 20 and $40^{\circ} \mathrm{N}$ in the lower and middle stratosphere. This might be an over-optimistic result, because it is due to small positive values at these locations that exist throughout the winter season. In this respect the climatology of probability of positive refraction index squared does not meet the criterion 4 in Table 1.

Further evidence to show the importance of $\frac{\partial}{\partial z} \bar{u}$ for vertical propagation of Rossby waves can be provided by calculating the normalized vertical component of the EliassenPalm (EP) flux. Figure 5 shows that the normalized vertical component of EP flux has a minimum at the tropopause, indicating that upward penetration of waves is suppressed by the negative values above tropopause heights as suggested by $\mathrm{Hu}$ and Tung (2002). Sensitivity of $m_{k, l}^{2}(y, z)$ to $\bar{u}$ can be studied by comparing the values of $a^{2} \frac{\overline{q_{\phi}}}{\bar{u}}$ and $a^{2} \frac{\overline{q_{\phi}}}{10 \mathrm{~ms}^{-1}}$. Fig- ure 6 shows the climatology of $a^{2} \frac{\overline{q_{\phi}}}{\bar{u}}$ and $a^{2} \frac{\overline{q_{\phi}}}{10 \mathrm{~ms}^{-1}}$ for DJF in the Northern Hemisphere. The subpolar maxima of $a^{2} \frac{\overline{q_{\phi}}}{\bar{u}}$ in the troposphere are not related to small values of the zonal wind at these regions, since by taking away the $\bar{u}$, the maxima are shifted to subtropics $\left(25-40^{\circ} \mathrm{N}\right)$. This implies that small values of $\bar{u}$ rather than $\frac{\partial}{\partial z} \bar{u}$ at subpolar regions cause the maxima of $m_{k, l}^{2}(y, z)$ at these regions.

\section{Probability of favorable propagation condition for Rossby waves}

A long standing issue in the interpretation of $m_{k, l}^{2}(y, z)$ is its vagueness. As suggested by Matsuno (1970), large waves tend to propagate in regions of positive vertical wave number $m_{k, l}^{2}(y, z)$ while they may be trapped in vertical direction where $m_{k, l}^{2}(y, z)<0$. Here (in the light of fuzzy sets and logic), we attempt to address the modeling of such vagueness. Fuzzy logic is a mathematical method for answering questions with imprecise information (such as very large or very small vertical wave number), it deals with reasoning that is approximate rather than fixed and precise. The basic approach is to assign a value between zero and one to describe the range between the upper and lower limit. The upper and lower limits refer to the maximum and minimum values of any variable. Within these limits fuzzy logic assigns a membership value function (MVF) (Zadeh, 1965; Novak et al., 1999).

Here we assume that instead of each of the individual $m_{k, l}^{2}(y, z, t)$ contributing equally to the time-mean $m_{k, l}^{2}(y, z)$, some $m_{k, l}^{2}(y, z, t)$ contribute more than others. In this way, we distinguish between small positive and very large positive values to let very large positive values influence the final result more than small positive values. In this way classes or sets whose boundaries are not sharp will be introduced. We introduce $\mu_{\mathrm{Ro}}(y, z, t)$ as the Rossby wave MVF which provides MPDF and estimate the probability of favorable propagation conditions of Rossby wave $\operatorname{Pr}_{\mathrm{Ro}}(y, z)$, as a function of latitude and height. We also provide the physical basis of the proposed method. For a detailed discussion of membership value function (MVF), see the Appendix A.

The advantage of our analysis over the traditional analysis of the vertical wave number is that without any reduction in the information due to cancellation of negative and positive values of the vertical wave number squared, we estimate the likeliness for planetary waves to propagate from one region to another at any time, altitude and latitude.

In Fig. 7 the black curve shows the MVF used in the calculation of favorable propagation condition of Rossby waves. For the negative $m_{k, l}^{2}(y, z, t)$ region (part a) this function suggests that the rate of attenuation is very high and therefore wave propagation is prohibited in this region. Since our method is still based upon the linear wave theory, we assume a linear relationship between the magnitude of the 
$(\mathrm{k}, \mathrm{l})=(1,1)$

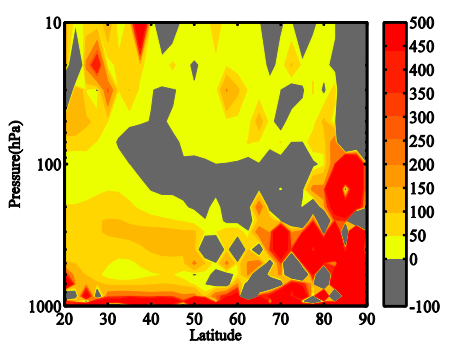

$(\mathrm{k}, \mathrm{l})=(2,1)$

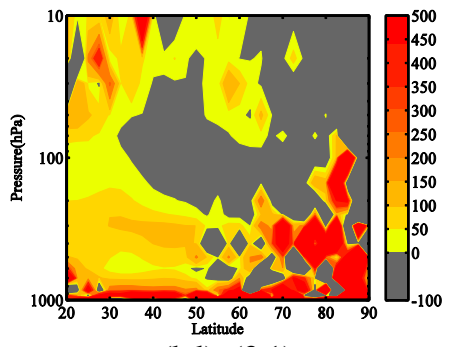

$(\mathrm{k}, \mathrm{l})=(3,1)$

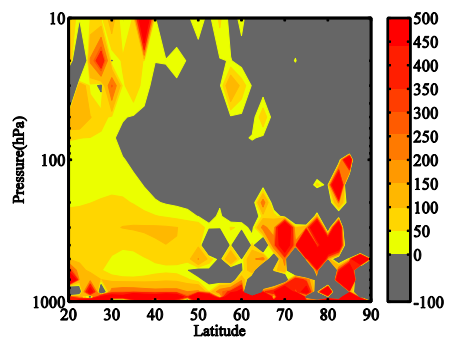

$(\mathrm{k}, 1)=(1,2)$

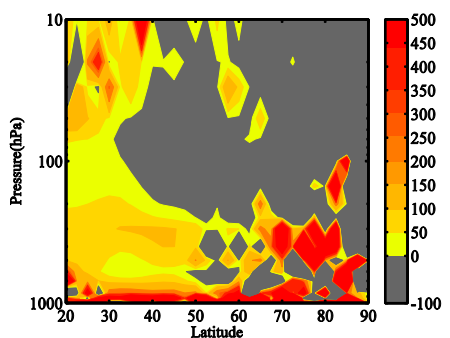

$(\mathrm{k}, \mathrm{l})=(2,2)$

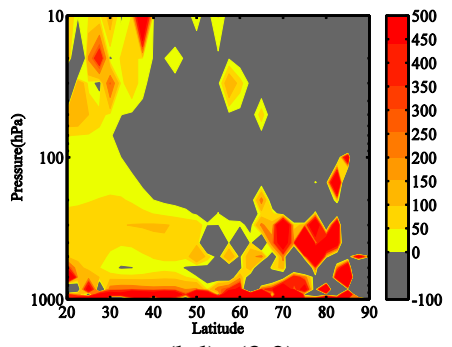

$(\mathrm{k}, \mathrm{l})=(3,2)$

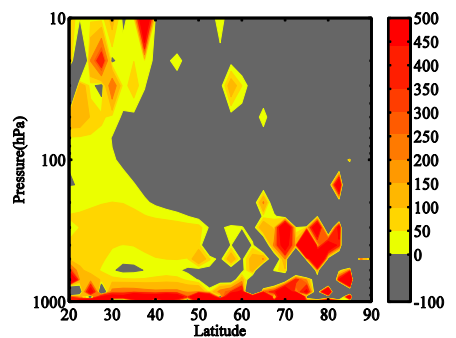

$(\mathrm{k}, \mathrm{l})=(1,3)$

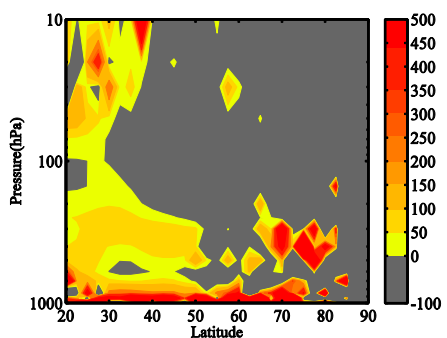

$(\mathrm{k}, \mathrm{l})=(2,3)$

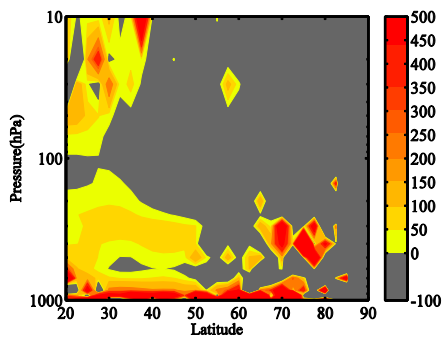

$(\mathrm{k}, \mathrm{l})=(3,3)$

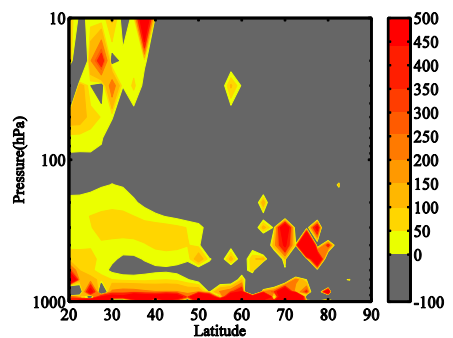

Figure 2. Climatology of vertical wave number squared $\left(a^{2} m_{k, l}^{2}(y, z)\right)$ of 50 winters (1961-2010) in the Northern hemisphere. Regions with negative $a^{2} m_{k, l}^{2}(y, z)$ are shaded in gray.

$m_{k, l}^{2}(y, z, t)$ and the probability of favorable propagation conditions for positive $m_{k, l}^{2}(y, z, t)$ in a way that the higher the values of the $m_{k, l}^{2}(y, z, t)$ the chances of propagation for the Rossby waves increases linearly (part b). Large values of the $m_{k, l}^{2}(y, z, t)$ occur near the critical line where zonal mean zonal wind approaches zero $\left(\bar{u}<0.5 \mathrm{~ms}^{-1}\right.$ in this study). This region is also not favorable for Rossby wave propagation since at this region the linear wave theory breaks down and waves start to break and the waves are absorbed (part c). The region where vertical wave number squared is larger than 600 is not favorable for wave propagation. At these regions the zonal mean zonal wind approaches zero. This condition often happens in the upper troposphere/lower stratosphere where westerlies become weak in the winter season near the Arctic. Therefore most of the differences between Figs. 4 and 8 for Rossby wave $(1,1)$ at the above-mentioned regions can be associated with setting $\mu_{\text {Ro }}$ to zero for $m_{k, l}^{2}>600$. In the study of Li et al. (2007) the effect of the critical line on Rossby wave propagation is neglected since all the positive values of the $m_{k, l}^{2}(y, z, t)$ are regarded as small and very large positive values of the $m_{k, l}^{2}(y, z, t)$ are equally favorable places for wave propagation. In fact very high values of the $m_{k, l}^{2}(y, z, t)$ are not necessarily favorable conditions for the Rossby wave propagation. In this study the $m_{k, l}^{2}(y, z, t)$ higher than 600 is considered as the critical line region, obtained from the climatology of the vertical wave number when $\bar{u}<0.5 \mathrm{~ms}^{-1}$. As we will show, this function gives us an improved picture of planetary wave propagation conditions in climatologies. Higher values of $\operatorname{Pr}_{\mathrm{Ro}}(y, z)$ provide a window of opportunity for planetary waves to propagate at any latitude and height. Likewise, smaller values of $\operatorname{Pr}_{\mathrm{Ro}}(y, z)$ demonstrate the places where Rossby waves are likely to be trapped in the vertical direction. The sensitivity of $\operatorname{Pr}_{\mathrm{Ro}}(y, z)$ values to the shape of the MVF function is discussed in Appendix A.

\section{Results and discussions}

Figure 8 demonstrates the climatology of probability of favorable propagation conditions of Rossby waves for zonal 
$(\mathrm{k}, \mathrm{l})=(1,1)$

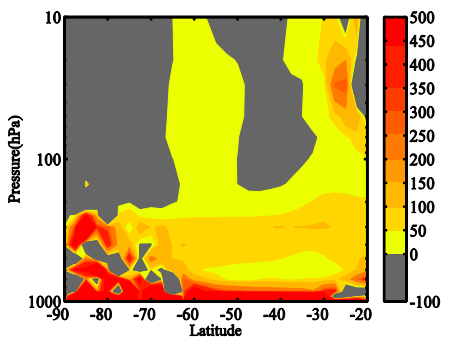

$(\mathrm{k}, 1)=(2,1)$

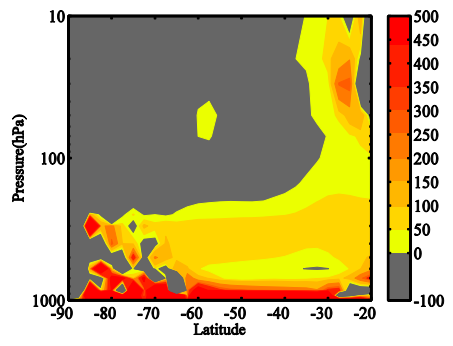

$(\mathrm{k}, \mathrm{l})=(3,1)$

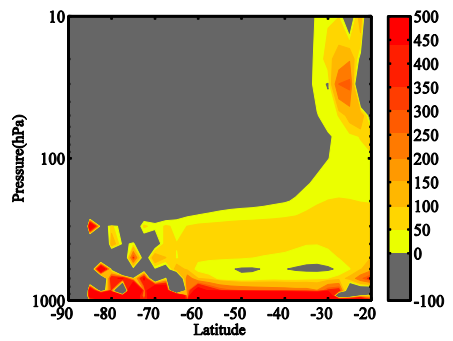

$(\mathrm{k}, 1)=(1,2)$

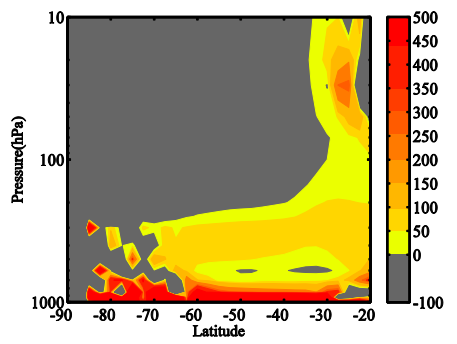

$(\mathrm{k}, \mathrm{l})=(2,2)$

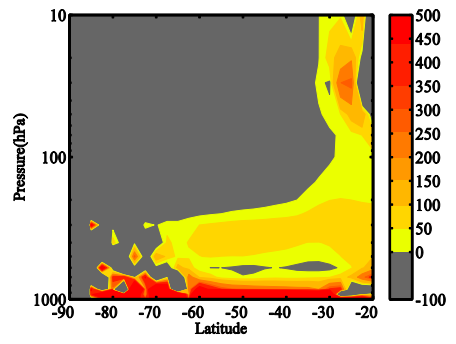

$(\mathrm{k}, \mathrm{l})=(3,2)$

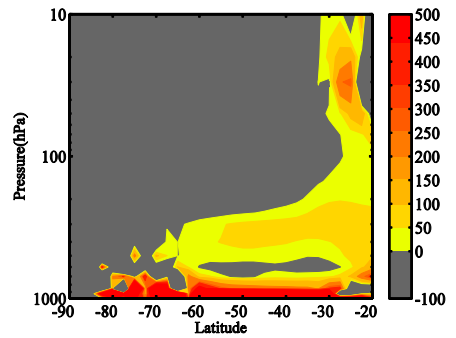

$(\mathrm{k}, \mathrm{l})=(1,3)$

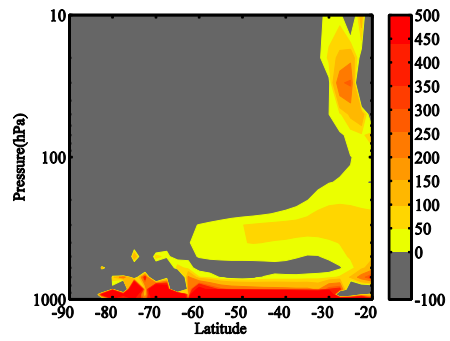

$(\mathrm{k}, \mathrm{l})=(2,3)$

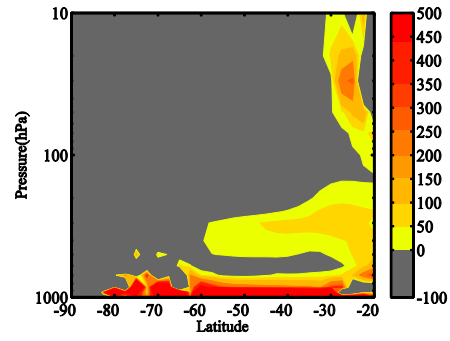

$(\mathrm{k}, \mathrm{l})=(3,3)$

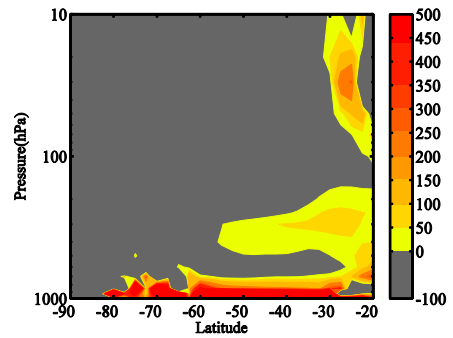

Figure 3. Climatology of vertical wave number squared $\left(a^{2} m_{k, l}^{2}(y, z)\right)$ of 50 winters $(1961-2010)$ in the Southern Hemisphere. Regions with negative $a^{2} m_{k, l}^{2}(y, z)$ are shaded in gray.

$(\mathrm{k}, \mathrm{l})=(1,1)$

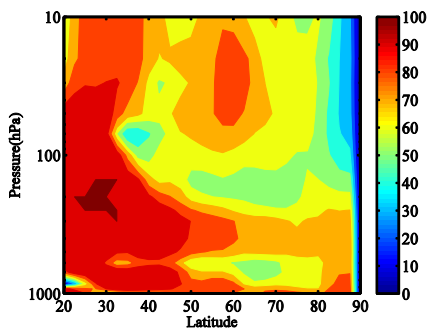

$(\mathrm{k}, \mathrm{l})=(1,2)$

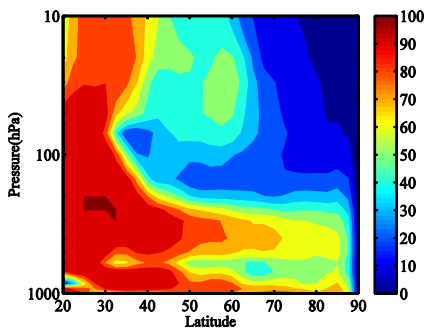

$(\mathrm{k}, \mathrm{l})=(1,3)$

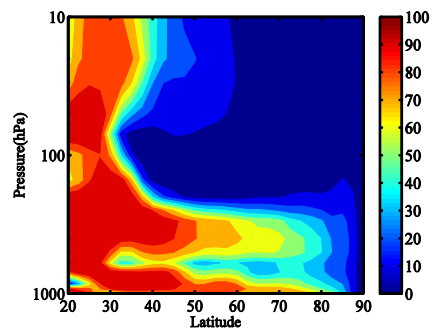

Figure 4. Probability of positive vertical wave number squared for Northern Hemisphere wintertime for wave $(1,1),(1,2)$ and $(1,3)$.

wave numbers $(k=1,2,3)$ and meridional wave numbers $(l=1,2,3)$ for the Northern Hemisphere winter season. The most common feature for all waves is their rather large probability to propagate in the troposphere (below $200 \mathrm{hPa}$ ) in winter season. It is also evident that the most favorable propagation condition is in the lower troposphere of the mid-latitude region. The values of Fig. 8 are independent of Rossby wave generation and explain how the waves, when generated, would propagate given the structure of the mean flow.
However, the regions of highly favorable Rossby wave propagation and source region for wave generation (asymmetries at the surface, land-sea contrasts, and sea surface temperature asymmetries) are coincident. It is also clear that longer waves have more opportunity to penetrate the stratosphere.

Karoly and Hoskins (1982) by using ray tracing technique from geometrical optics and wave propagation in a slowly varying medium, showed that wave rays which are parallel to the group velocity vector tend to refract toward large vertical 


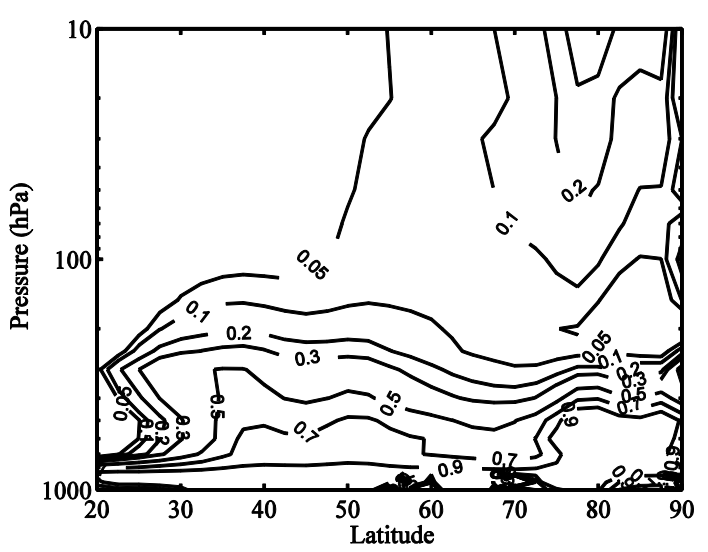

Figure 5. Climatology of vertical component of EP flux normalized by vertical component of EP flux at $850 \mathrm{hPa}$ for DJF at Northern Hemisphere. Discontinuity of this quantity at the tropopause heights indicates the strong suppression of wave penetration from troposphere into the stratosphere at lower stratosphere. Chen and Robinson (1992) discuss the importance of the abrupt change of the buoyancy frequency at the tropopause level for the suppression of the upward wave propagation.

wave number squared. They also found that Rossby waves have a tendency to propagate along great circles and most of the upward propagation of Rossby waves will be refracted toward the equator (even if the vertical wave number squared were positive at all heights in their study). Similar to this theory, we also found a channel or waveguide of large probability of favorable propagation condition for Rossby waves. The strong westerlies act as a waveguide of Rossby waves and direct them vertically through the tropopause and allow them to penetrate to higher altitudes from their source region (troposphere). These areas are south of $40^{\circ} \mathrm{N}$ in winter of the Northern Hemisphere for large waves and are indicated by $\operatorname{Pr}_{\mathrm{Ro}}(y, z)>50 \%$.

The study of Karoly and Hoskins (1982) also revealed that Rossby waves tend to propagate on the edges of strong westerlies and avoid penetrating through the jet. This fact is also clear in our results, where north of $60^{\circ} \mathrm{N}$ and above $200 \mathrm{hPa}$, the probability of favorable condition for Rossby waves show relatively smaller values, compared to similar altitude ranges between 30 and $50^{\circ} \mathrm{N}$. The maxima south of $40^{\circ} \mathrm{N}$ at $100 \mathrm{hPa}$ in the mPDF shows that the region is favorable for wave propagation. At the same region, the vertical component of the EP fluxes have small magnitudes. However as shown in Li et al. (2007) the horizontal component of EP fluxes has large values at this region (Fig. 5e in the study of Li et al., 2007). Since the current study concentrates only on the vertical wave propagation, not all aspects of Fig. 5 can be directly compared with Fig. 8 . The same climatologies as Fig. 8 are presented in Fig. 9 for the Southern Hemisphere. Similar to the Northern Hemisphere, all large-scale waves have a rather large chance to propagate in the troposphere in winter. It can be seen that the larger the waves, the higher the probability of favorable conditions for them to propagate upward.

Figure 10 demonstrates the differences between probability of positive vertical wave number (calculated by PDFs) and probability of favorable propagation condition of Rossby waves (calculated by mPDFs) for Northern Hemisphere wintertime for wave $(1,1),(1,2)$ and $(1,3)$. The maximum difference is found at $20-40^{\circ} \mathrm{N}$ of the middle and upper troposphere which can reach to $50 \%$. This unsatisfactory result of the probability of positive vertical wave number is due to small positive values at these places which is consistent throughout the winter season. The area of maximum difference between $\operatorname{Pr}_{\mathrm{Ro}}(y, z)$ and probability of positive vertical wave number remains the same for all wave numbers at both Northern and Southern hemispheres (not shown).

As Figs. 8 and 9 show the most important difference between the Northern and Southern hemisphere occurs in the high latitudes of the stratosphere, where in the Northern Hemisphere, zonal wave number $=1$ has a good opportunity to propagate $\left(\operatorname{Pr}_{\mathrm{Ro}}(y, z)>40 \%\right)$, while in the Southern Hemisphere it has a rather poorer chance to propagate. This is consistent with the theoretical explanation of the vertical propagation of Rossby waves from the troposphere to the stratosphere by Charney and Drazin (1961). The zonal mean zonal wind should be weaker than a critical strength for upward propagation of Rossby waves. The strong stratospheric winter polar vortex of the Southern Hemisphere will block and reflect wave activity. The critical strength depends on the scale of the wave and is not a function of the background zonal regime.

A significant piece of information which is lost from the time mean of $m_{k, l}^{2}(y, z)$ is the role of meridional wave numbers on the wave propagation conditions. For instance in the Southern Hemisphere, the difference between the time mean of $m_{k, l}^{2}(y, z)$ for wave $(2,1),(2,2)$ and $(2,3)$ in the stratosphere (above $100 \mathrm{hPa}$ ) is not large which is one of the unsatisfactory results of time mean of $m_{k, l}^{2}(y, z)$. It is only in the light of $\operatorname{Pr}_{\mathrm{Ro}}(y, z)$ values that we can understand the impact of meridional wave numbers on the wave propagation in the stratosphere. Note that, at the same latitude range of the Southern Hemisphere, $\operatorname{Pr}_{\mathrm{Ro}}(y, z)$ values are as high as $45 \%$ for wave $(2,1)$ in mid-latitudes of stratosphere, while the $\operatorname{Pr}_{\mathrm{Ro}}(y, z)$ values reach to less than $5 \%$ for wave $(2,3)$.

\section{Usefulness and appropriateness of $\operatorname{Pr}_{\operatorname{Ro}}(y, z)$}

In order to test the appropriateness of the $\operatorname{Pr}_{\mathrm{Ro}}(y, z)$ in climatological studies of stationary planetary wave propagation, we further investigate the sensitivity of the $\operatorname{Pr}_{\mathrm{Ro}}(y, z)$ to different zonal flow regimes in the stratosphere. Following Castanheira and Graf (2003), we constructed two data sets based upon the strength of the westerlies in the lower stratosphere $(50 \mathrm{hPa})$ at $65^{\circ} \mathrm{N}$. According to the Charney and Drazin (1961) criterion, if the background flow is westerly 

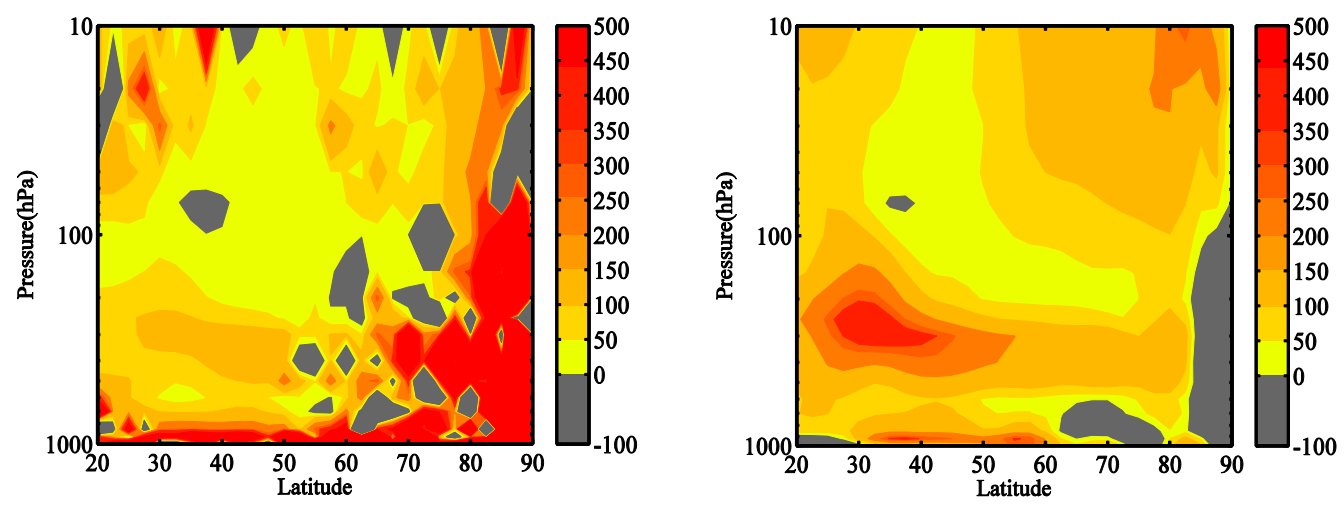

Figure 6. climatology of $a^{2} \frac{\overline{q_{\phi}}}{\bar{u}}$ (left) and $a^{2} \frac{\overline{q_{\phi}}}{10}$ (right) for DJF in the Northern Hemisphere.

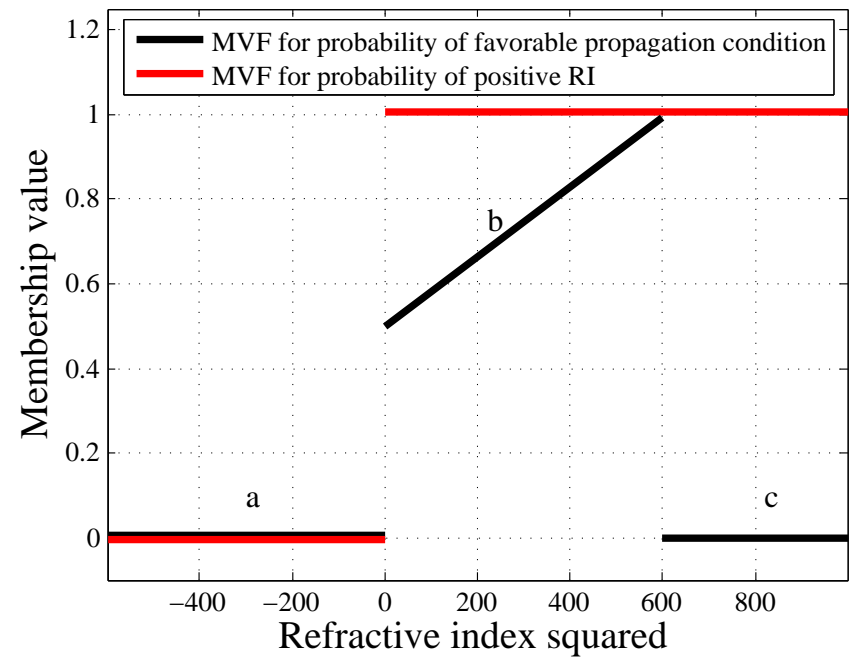

Figure 7. MVF used in the calculation of favorable propagation condition of Rossby waves (black curve). Red lines show MVF for calculating probability of positive vertical wave number which are used by Li et al. (2007). In their study the effect of the critical layer (part c) is not considered.

and smaller than the latitude and wave number dependent critical Rossby velocity, the planetary waves can penetrate from the troposphere into the stratosphere, otherwise wave reflection occurs and tropospheric flow may be modified. strong vortex regime $(\mathrm{SVR})$ is identified when $\bar{u}_{50}\left(65^{\circ} N\right)>$ $20 \mathrm{~ms}^{-1}$ and weak vortex regime (WVR) is considered when $0<\bar{u}_{50}\left(65^{\circ} \mathrm{N}\right)<10 \mathrm{~ms}^{-1}$, where $\bar{u}_{50}\left(65^{\circ} \mathrm{N}\right)$ is the $50 \mathrm{hPa}$ zonal mean zonal wind at $65^{\circ} \mathrm{N}$. The $20 \mathrm{~ms}^{-1}$ threshold reflects the critical Rossby velocities $\left(20 \mathrm{~ms}^{-1}\right)$ for $\mathrm{ZWN}=1$ for a climatological Northern Hemisphere zonal wind profile. The WVR events do not correspond to the sudden stratospheric warmings (SSWs) in the current study. Since during SSWs the linear wave theory breaks down and waves start to break and the waves are absorbed, the vertical wave number and probability of the favorable wave propagation (both are
Table 2. Periods of polar vortex regimes lasting for at least 30 consecutive days in DJF; left: strong vortex regime. Right: weak vortex regime.

\begin{tabular}{rr|rr}
\hline \multicolumn{2}{c|}{ Strong vortex regime (SVR) } & \multicolumn{2}{c}{ Weak vortex regime (WVR) } \\
Starting date & Ending date & Starting date & Ending date \\
\hline 20 Dec 1961 & 20 Feb 1962 & 20 Dec 1968 & 27 Jan 1969 \\
24 Dec 1963 & 28 Feb 1964 & 28 Dec 1984 & 13 Feb 1985 \\
3 Jan 1967 & 28 Feb 1967 & 9 Dec 1998 & 11 Jan 1999 \\
1 Dec 1975 & 28 Feb 1976 & 2 Jan 2004 & 28 Feb 2004 \\
1 Dec 1987 & 14 Jan 1988 & & \\
16 Dec 1988 & 17 Feb 1989 & & \\
17 Dec 1989 & 28 Feb 1990 & & \\
1 Dec 1991 & 18 Jan 1992 & & \\
5 Dec 1992 & 11 Feb 1993 & & \\
1 Dec 1994 & 18 Jan 1995 & & \\
7 Dec 2004 & 21 Feb 2005 & \\
30 Dec 2006 & 26 Feb 2007 & \\
23 Dec 2007 & 13 Feb 2008 & \\
\hline
\end{tabular}

based on the linear wave theory) have limitations for studying the wave propagation during SSWs.

Table 2 demonstrates the periods of different polar vortex regimes that last for at least 30 consecutive days in DJF. Since in DJF the stratospheric flow consists of strong westerlies (in the absence of vertical wave propagation), the number of SVR events is higher than WVR events. The results of $m_{k, l}^{2}(y, z)$ and $\operatorname{Pr}_{\mathrm{Ro}}(y, z)$ for WVR and SVR for wave $(1,1)$ are presented in Fig. 11. It is found that in comparison to climatologies (Fig. 8) both WVR and SVR show similar patterns. However, the waveguide at mid-latitudes is much narrower in SVR than WVR. In addition, the average values of $\operatorname{Pr}_{\mathrm{Ro}}(y, z)$ in the stratosphere are greater in WVR than SVR. These results show that planetary waves have more of a chance to penetrate and force the stratosphere in WVR than SVR. In other words, values of $\operatorname{Pr}_{\mathrm{Ro}}(y, z)$ are sensitive to stratospheric westerlies and are consistent with the general knowledge about planetary wave propagation from the tropo- 
$(\mathrm{k}, \mathrm{l})=(1,1)$

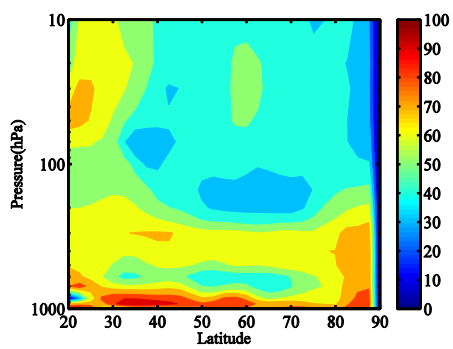

$(\mathrm{k}, \mathrm{l})=(2,1)$

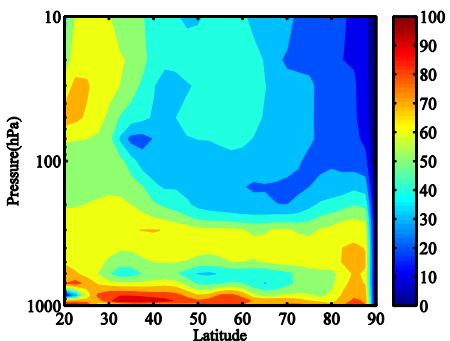

$(\mathrm{k}, \mathrm{l})=(3,1)$

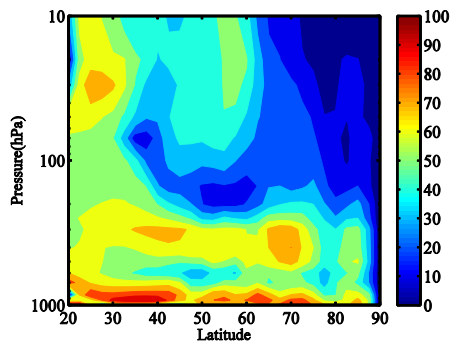

$(\mathrm{k}, \mathrm{l})=(1,2)$

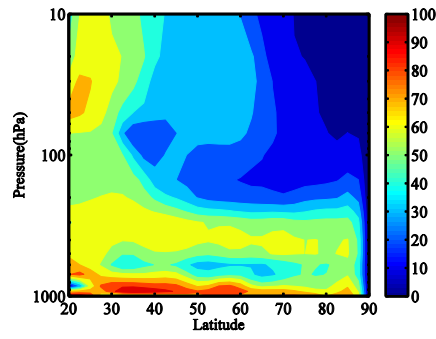

$(\mathrm{k}, \mathrm{l})=(2,2)$

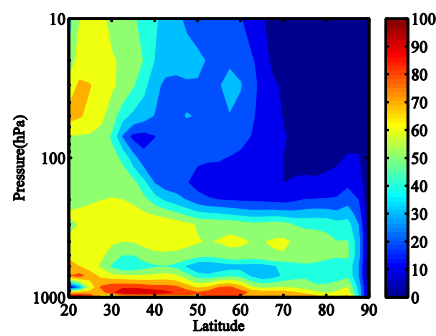

$(\mathrm{k}, \mathrm{l})=(3,2)$

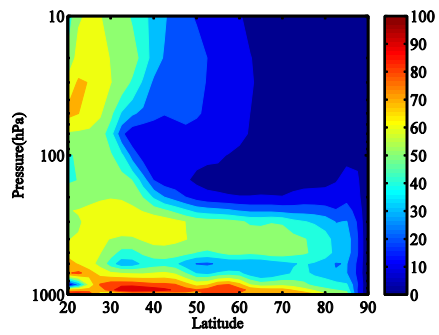

$(\mathrm{k}, \mathrm{l})=(1,3)$

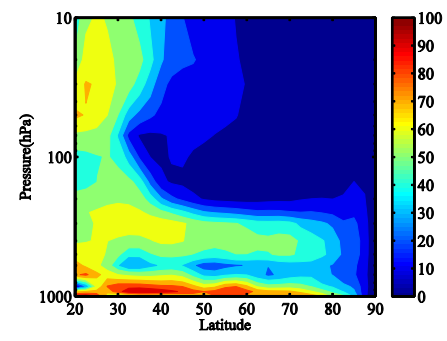

$(\mathrm{k}, \mathrm{l})=(2,3)$

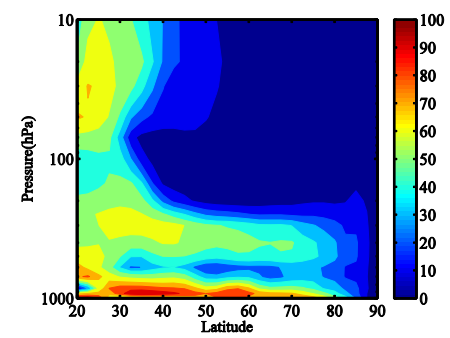

$(\mathrm{k}, \mathrm{l})=(3,3)$

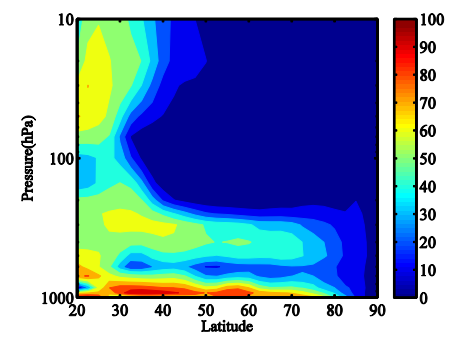

Figure 8. Probability of favorable propagation condition for Rossby waves derived from 50 winters (1961-2010) in the Northern Hemisphere. The higher the values, the more convenient it is for planetary waves to propagate to that region. In contrast, planetary waves are likely to be trapped in the vertical direction when the value of this quantity is small.

sphere to the stratosphere. An enhancement of wave propagation northward of $70^{\circ} \mathrm{N}$ in the lower stratosphere and a slight reduction in the favorability of wave propagation between 50 and $70^{\circ} \mathrm{N}$ in the stratosphere are found for WVR. On the other hand it can be seen that due to the high level of noisiness the interpretation of the difference of $m_{k, l}^{2}(y, z)$ between WVR and SVR is very difficult. Since the highest difference in the favorability of wave propagation between WVR and SVR occurs northward of $50^{\circ} \mathrm{N}$ in the stratosphere, we further calculate the difference in the vertical component of EP flux between WVR and SVR in this region (Fig. 12). An enhancement of vertical EP flux is obtained northward of $65^{\circ} \mathrm{N}$ in the lower stratosphere during WVR while a decrease in this quantity is obtained southward of this region in the middle and upper stratosphere. By comparing the differences of $m_{k, l}^{2}(y, z), \operatorname{Pr}_{\mathrm{Ro}}(y, z)$ and vertical component of EP flux during WVR and SVR, it can be seen that the pattern of differences between $\operatorname{Pr}_{\mathrm{Ro}}(y, z)$ and vertical component of EP flux are similar. Therefore, based upon these analyses, we sug- gest that this diagnostic tool can be useful for studying the propagating properties of the planetary waves.

\section{Conclusions}

Climatological values of the time mean of the vertical wave number squared derived from 50 winters (1961-2010) of both Northern and Southern hemispheres are calculated to show several problematic features of this important quantity in climatologies. In order to improve these unsatisfactory results, we introduced probability density functions (PDFs) of positive vertical wave number as a function of zonal and meridional wave numbers. We also compared this quantity with a modified set of PDFs (mPDFs) and demonstrated their superior performance compared to the climatological mean of vertical wave number and the original PDFs. Without any reduction in the information, $\operatorname{Pr}_{\mathrm{Ro}}(y, z)$ estimates the likeliness for stationary Rossby waves to propagate from one region to another at any time, altitude and latitude in a clima- 
$(\mathrm{k}, \mathrm{l})=(1,1)$

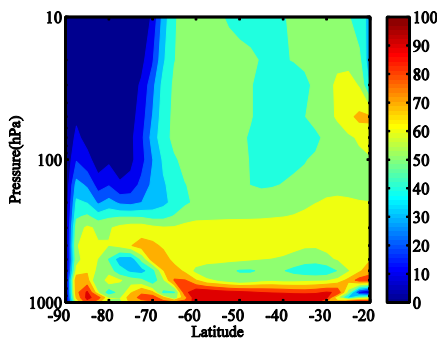

$(\mathrm{k}, \mathrm{l})=(2,1)$

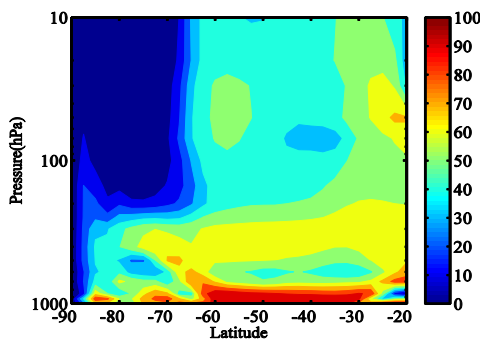

$(\mathrm{k}, \mathrm{l})=(3,1)$

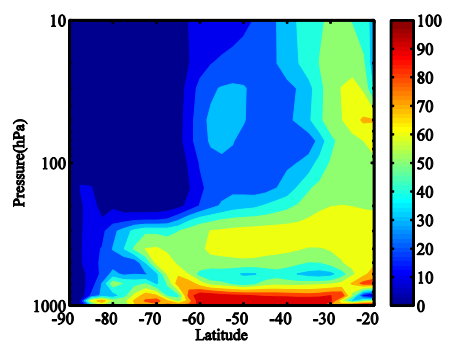

$(\mathrm{k}, 1)=(1,2)$

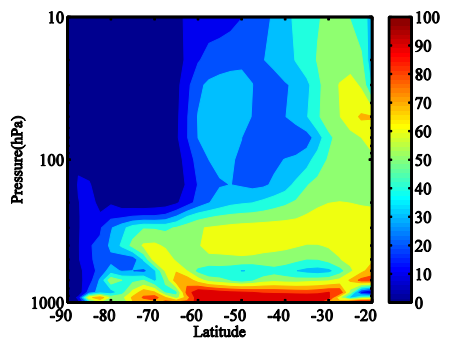

$(\mathrm{k}, 1)=(2,2)$

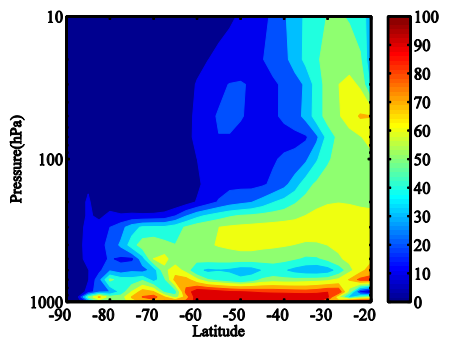

$(\mathrm{k}, \mathrm{l})=(3,2)$

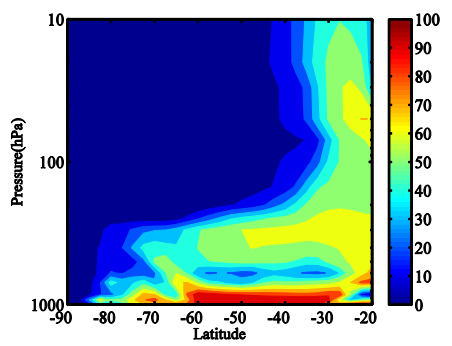

$(\mathrm{k}, \mathrm{l})=(1,3)$

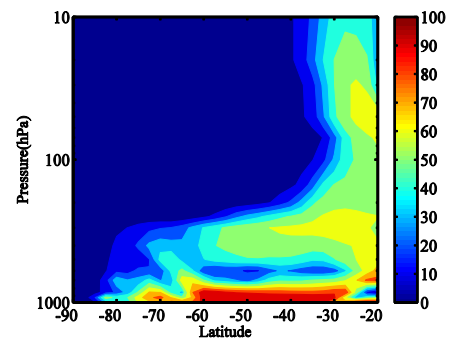

$(\mathrm{k}, \mathrm{l})=(2,3)$

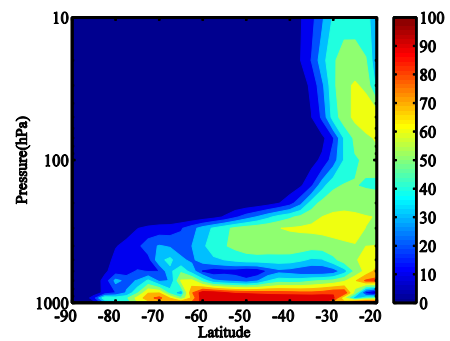

$(\mathrm{k}, 1)=(3,3)$

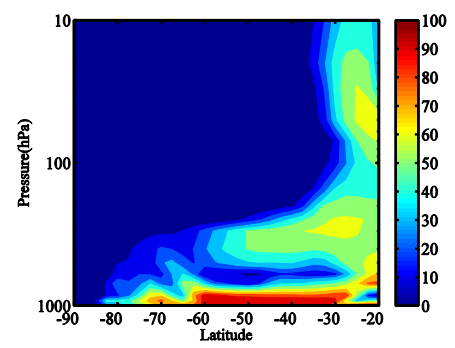

Figure 9. The same as Fig. 8 but for Southern Hemisphere wintertime.

$(\mathrm{k}, \mathrm{l})=(1,1)$

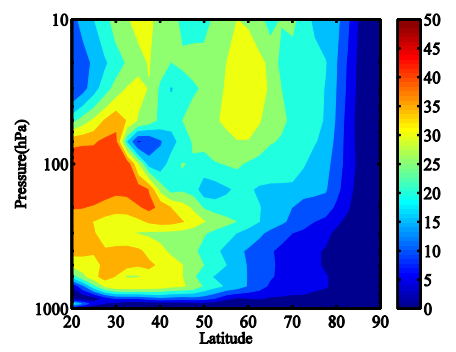

$(\mathrm{k}, \mathrm{l})=(1,2)$

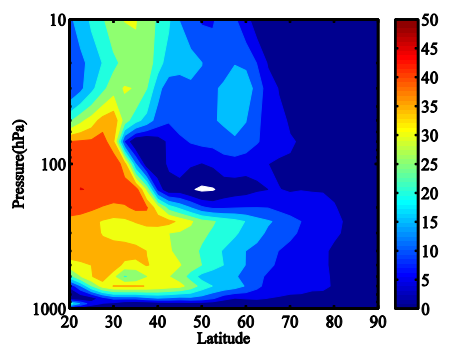

$(\mathrm{k}, \mathrm{l})=(1,3)$

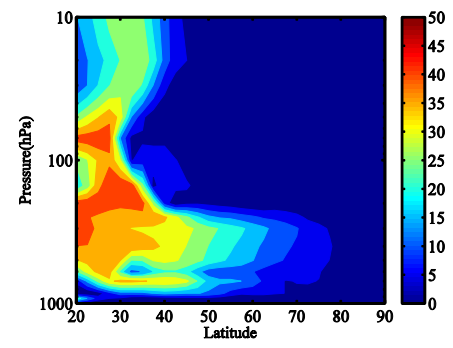

Figure 10. The differences between the probability of positive vertical wave number squared and the probability of favorable propagation condition of stationary Rossby waves.

tological sense. The higher the $\operatorname{Pr}_{\mathrm{Ro}}(y, z)$ the easier it is for planetary waves to propagate. Smaller values of $\operatorname{Pr}_{\mathrm{Ro}}(y, z)$ demonstrate the places where Rossby waves are likely to be trapped in the vertical direction. It is also found that by using this quantity one can easily study the difference in stationary Rossby wave propagation between different meridional wave numbers without the difficulty of the interpretation of the noisy structure of the time mean vertical wave number.
Our diagnostic tool is also capable of demonstrating the enhancing influence of positive vertical shear of zonal wind and impeding influence of negative vertical shear of zonal wind on stationary Rossby wave propagation from the troposphere to the stratosphere. The better performance of the mPDF suggests that relatively small but positive numbers of the vertical wave number squared play an important role to offer a favorite propagating condition for planetary waves in 

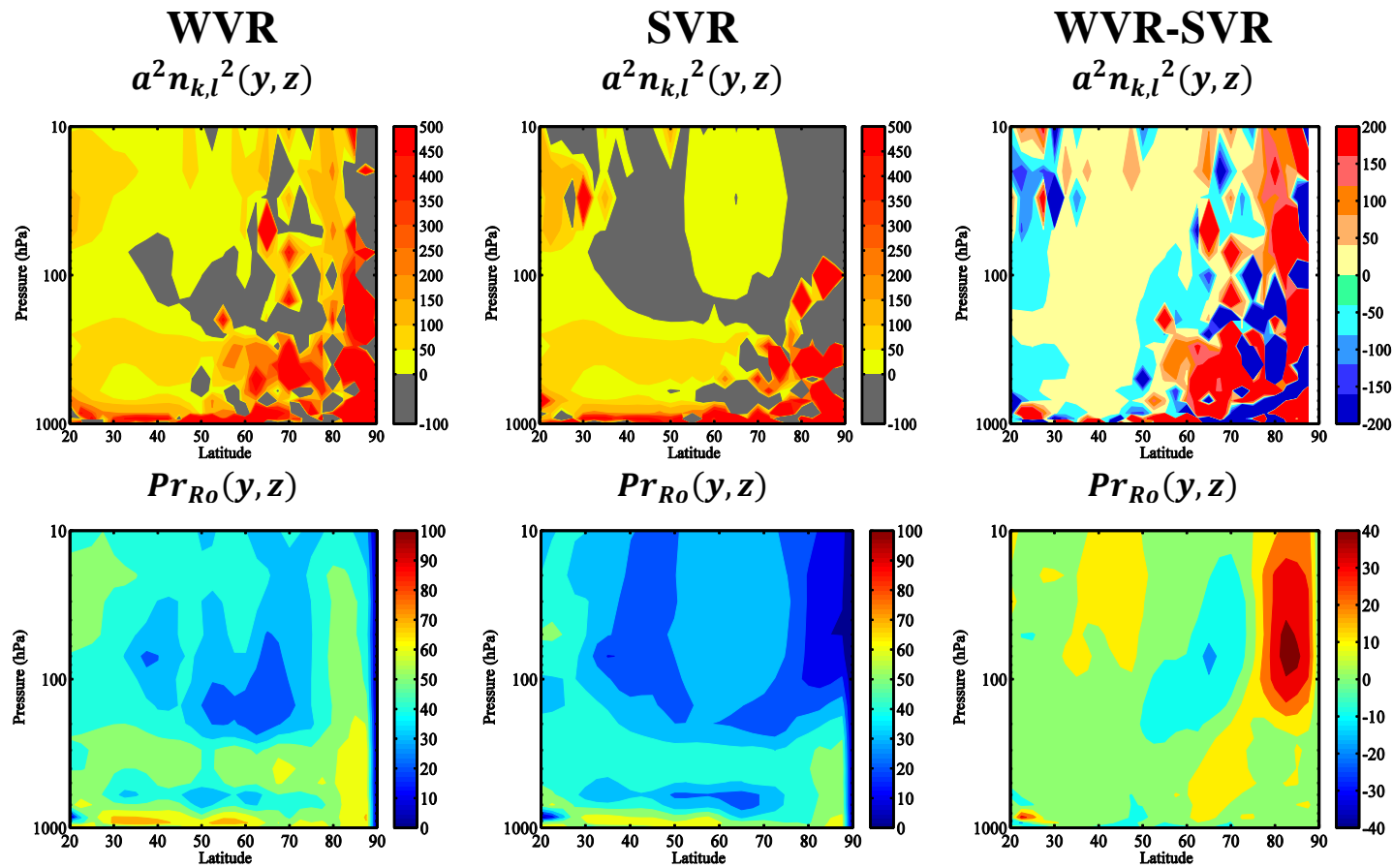

Figure 11. $a^{2} m_{k, l}^{2}(y, z)$ (first row) and $\operatorname{Pr}_{\mathrm{Ro}}(y, z)$ (second row) during WVR and SVR.

WVR

$\mathbf{F}_{\mathbf{z}}$

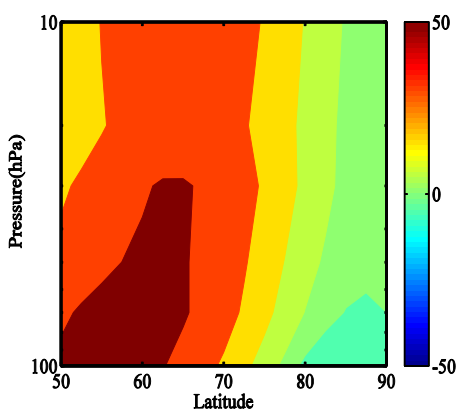

SVR

$\mathbf{F}_{\mathbf{z}}$

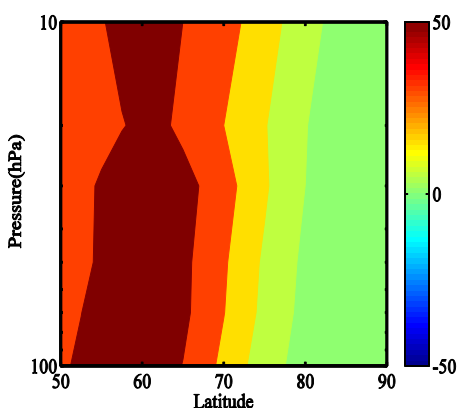

WVR-SVR

$\mathbf{F}_{\mathbf{z}}$

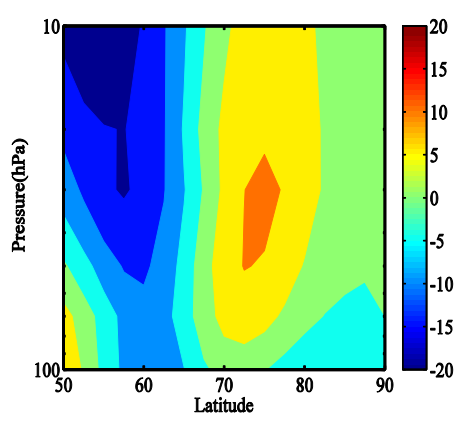

Figure 12. Same as Fig. 11 but restricted to the 100-10 hPa range for the vertical component of EP flux. The values are divided by $10^{5}$. Since the highest differences in the $m_{k, l}^{2}(y, z)$ and $\operatorname{Pr}_{\mathrm{Ro}}(y, z)$ between WVR and SVR are in the high latitude stratosphere the vertical component of EP fluxes are shown in this region.

the stratosphere. This diagnostic tool successfully shows that for WVR there is more space for the vertical propagation of Rossby waves from the troposphere to the stratosphere. In contrast, SVR tend to block and reflect vertical propagation of stationary Rossby waves. It is also worthwhile mentioning that both the vertical wave number and probability of the favorable wave propagation are still qualitative tools to study the vertical propagation of Rossby waves from the troposphere to the stratosphere. Since our diagnostic tool is consistent with the theoretical understanding of vertical propagation of Rossby waves from the troposphere to the strato- sphere, we suggest that this diagnostic tool has the capacity to be used in assessing planetary wave propagation conditions in climate models.

\section{Data availability}

The NCEP/NCAR data set is publicly available at http:// www.esrl.noaa.gov/psd. 


\section{Appendix A:}

The probability of favorable propagation condition of Rossby waves $\operatorname{Pr}_{\mathrm{Ro}}(y, z)$ can be written as

$\operatorname{Pr}_{\mathrm{Ro}}(y, z)=\frac{\sum_{t=1}^{n} \mu_{\mathrm{Ro}}(y, z, t)}{\sum_{t=1}^{n} t} \times 100$,

where $\mu_{\mathrm{Ro}}(y, z, t)$ as modified set of PDFs (mPDFs) is defined as:

$\mu_{\mathrm{Ro}}=$

$\left\{\begin{array}{l}0 \text { if } m_{k, l}^{2} \leq 0, \\ \left(8.3 \times 10^{-4} \times m_{k, l}^{2}(y, z)\right)+0.5 \text { if } 0<m_{k, l}^{2}<600, \\ 0 \text { if } m_{k, l}^{2} \geq 600\end{array}\right.$

Here $8.3 \times 10^{-4}$ is the slope of line $b$ in Fig. 7. The variable $t$ is the time step and in the current study the daily mean values of the temperature and zonal wind are used in the calculations. In the study of Li et al. (2007) PDFs (red lines in the Fig. 7) are defined as

$\mu_{\mathrm{Ro}}=\left\{\begin{array}{l}0 \text { if } m_{k, l}^{2}<0, \\ 1 \text { if } m_{k, l}^{2}>0,\end{array}\right.$

In order to test the sensitivity of $\operatorname{Pr}_{\mathrm{Ro}}(y, z)$ to the shape of MVF, we evaluated the values of $\operatorname{Pr}_{\mathrm{Ro}}(y, z)$ for several potential MVFs. Figure A1 demonstrates the shapes of three MVFs that are used to calculate the values of $\operatorname{Pr}_{\mathrm{Ro}}(y, z)$. It can be seen from Fig. A2 (first row) that MVF1 gives unsatisfactory results above $200 \mathrm{hPa}$, where for wave $(3,3)$ we expect very low values of $\operatorname{Pr}_{\mathrm{Ro}}(y, z)$ poleward of $40^{\circ} \mathrm{N}$. This function (MVF1) neglects the fact that Rossby waves tend to quickly attenuate in low values of vertical wave number squared. The values of $\operatorname{Pr}_{\mathrm{Ro}}(y, z)$ can reach as high as $50 \%$ at these latitudes and altitudes. MVF2 and MVF3 also give unrealistic results where the values of $\operatorname{Pr}_{\mathrm{Ro}}(y, z)$ are too low in the stratosphere for all waves. These MVFs block all waves in the troposphere. Furthermore, they do not provide any waveguides in which Rossby waves can penetrate from troposphere to the stratosphere.

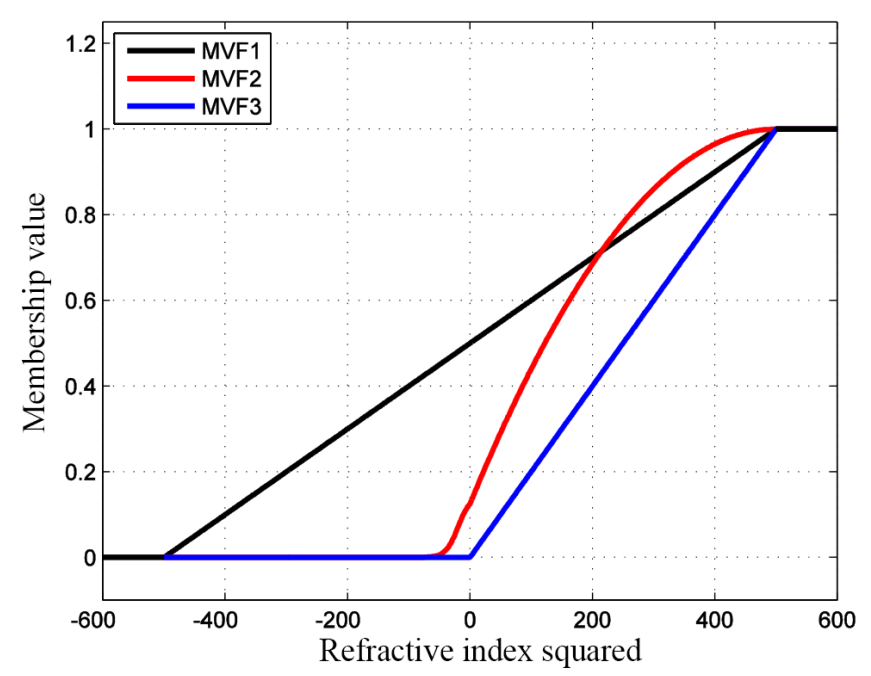

Figure A1. Shape of three MVFs that are used to calculate the values of $\operatorname{Pr}_{\mathrm{Ro}}(y, z)$. 
MVF1- $(\mathrm{k}, 1)=(1,1)$

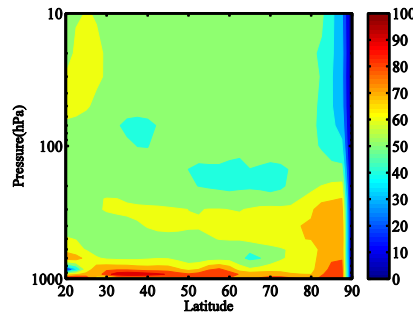

MVF2- $(\mathrm{k}, 1)=(1,1)$

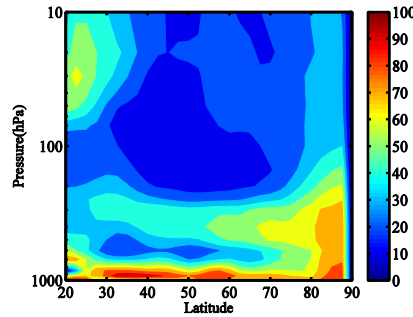

MVF3- $(\mathrm{k}, 1)=(1,1)$

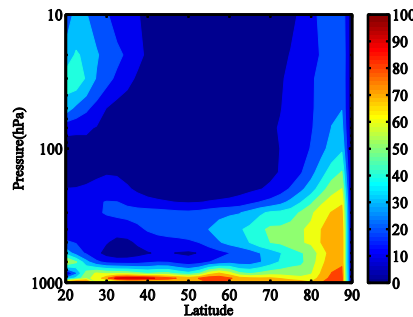

MVF1- $(\mathrm{k}, \mathrm{l})=(2,2)$

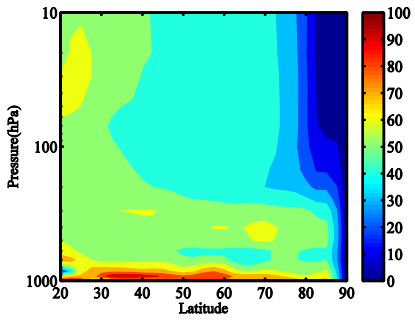

MVF2 $-(\mathrm{k}, 1)=(2,2)$

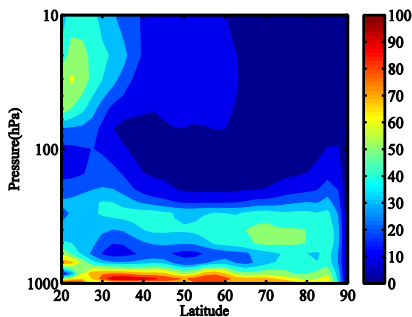

MVF3- $(\mathrm{k}, 1)=(2,2)$

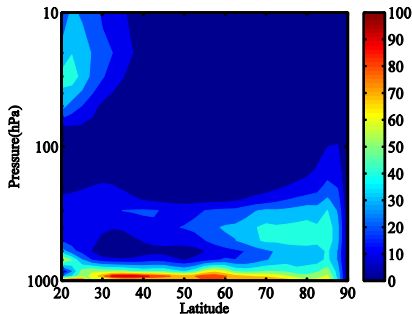

MVF1- $(\mathrm{k}, 1)=(3,3)$

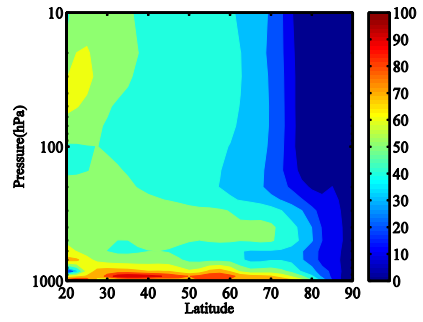

MVF2- $(\mathrm{k}, 1)=(3,3)$

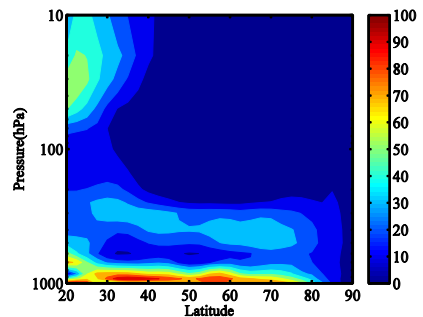

MVF3- $(\mathrm{k}, 1)=(3,3)$

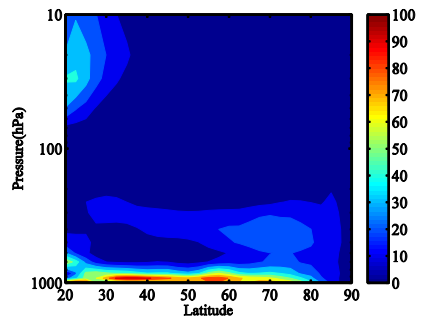

Figure A2. Probability of favorable propagation condition for Rossby waves derived from 50 winters (1961-2010) in the Northern Hemisphere based on different MVF values described in Fig. A1.
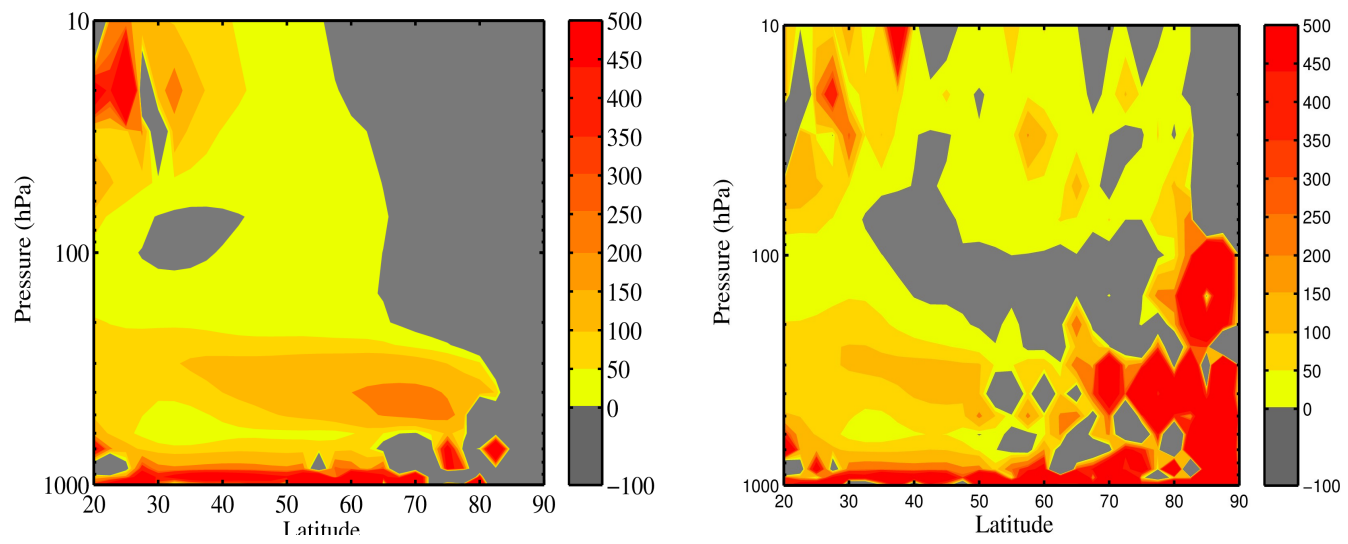

Figure A3. On the left the time-averaged zonal mean fields are used to calculate the vertical wave number squared (only for $(k, l)=(1,1))$. On the right the time mean of the vertical wave number is shown. It is clear that the vertical wave number derived from the time-averaged zonal mean fields has less noise than the time mean vertical wave number squared. We discuss this effect in more detail in the manuscript. Theoretically there are various ways in which one may reduce the level of noise in the time mean of the vertical wave number. The advantage of our proposed method is that it maps well and in a physical way on the list of criteria formulated in Table 1 . Alternatively one can use other statistical methods like truncated means or trimmed means to reduce the noisiness. 
Acknowledgements. Khalil Karami and Miriam Sinnhuber gratefully acknowledge funding by the Helmholtz Society within the Helmholtz Young Investigators group: Solar variability, climate, and the role of the mesosphere/lower thermosphere, project NWG-642. NCEP Reanalysis data provided by the NOAA/OAR/ESRL PSD, Boulder, Colorado, USA, from their Web site at http://www.esrl.noaa.gov/psd.

The article processing charges for this open-access publication were covered by a Research

Centre of the Helmholtz Association.

Edited by: M. Heimann

\section{References}

Andrews, D. G., Leovy, C. B., and Holton, J. R.: Middle Atmosphere Dynamics, Academic Press, San Diego, 1987.

Castanheira, J. M. and Graf, H.-F.: North Pacific-North Atlantic relationships under stratospheric control?, J. Geophys. Res., 108, 4036, doi:10.1029/2002JD002754, 2003.

Charney, J. G. and Drazin, P. G.: Propagation of planetary scale disturbances from the lower into the upper atmosphere, J. Geophys. Res., 66, 83-109, 1961.

Chen, P. C. and Robinson, W. A.: propagation of planetary waves between the troposphere and stratosphere, J. Atmos. Sci., 49, 2533-2545, 1992.

Dickinson, R. E.: Planetary Rossby waves propagating vertically through weak westerly wind wave guides, J. Atmos. Sci., 25, 984-1002, 1969.

Eliassen, A. and Palm, E.: On the transfer of energy in stationary mountain waves, Geofys. Publ., XXII, 1-23, 1961.

$\mathrm{Hu}$, Y. and Tung, K. K.: Interannual and decadal variations of planetary wave activity, stratospheric cooling, and northern hemisphere annular mode, J. Climate, 15, 1659-1673, 2002.

Huang, R. and Gambo, K.: The response of a Hemispheric MultiLevel Model Atmosphere to Forcing by Topography and Stationary Heat Sources (I) Foring by Topography, J. Meteorol. Soc. Jpn., 60, 78-92, 2002.
Kalnay, E., Kanamitsu, M., Kistler, R., Collins, W., Deaven, D., Gandin, L., Iredell, M., Saha, S., White, G., Woollen, J., Zhu, Y., Leetmaa, A., Reynolds, R., Chelliah, M., Ebisuzaki, W., Higgins, W., Janowiak, J., Mo, K. C., Ropelewski, C., Wang, J., Jenne, R., and Joseph, D.: The NCEP/NCAR 40-Year Reanalysis Project, Bull. Am. Meteorol. Soc., 77, 437-471, 1996.

Karoly, D. J. and Hoskins, B. J.: Three dimensional propagation of planetary waves, J. Meteorol. Soc. Jpn., 60, 109-122, 1982.

Li, Q., Graf, H.-F., and Giorgetta, M. A.: Stationary planetary wave propagation in Northern Hemisphere winter - climatological analysis of the refractive index, Atmos. Chem. Phys., 7, 183200, doi:10.5194/acp-7-183-2007, 2007.

Limpasuvan, V. and Hartmann, D. L.: Wave-maintained annular modes of climate variability, J. Climate., 13, 4414-4429, 2000.

Lin, B.: The behavior of winter stationary planetary waves forced by topagraphy and diabatic heating, J. Atmos. Sci., 39, 1206-1226, 1982.

Matsuno, T.: Vertical propagation of stationary planetary waves in the winter Northern Hemisphere, J. Atmos. Sci., 27, 871-883, 1970 .

Mukougawa, H. and Hirooka, T.: Predictability of stratospheric sudden warming: A case study for 1998/99 winter, Mon. Weather Rev., 132, 1764-1776, 2004.

Novak, V., Perfilieva, I., and Mockor, J.: Mathematical principles of fuzzy logic, Kluwer Academic Publishers, Springer US, 1999.

Randel, W. J.: The seasonal evolution of planetary waves in the southern hemisphere stratosphere and troposphere, Q. J. Roy. Meteor. Soc., 114, 1385-1409, 1988.

Smith, A. R.: Stationary waves in the winter stratosphere: Seasonal and interannual variability, J. Atmos. Sci., 40, 245-261, 1983.

Sun, C. and Li, J.: Space-Time Spectral Analysis of the Southern Hemisphere Daily 500-hPa Geopotential Height, Mon. Weather Rev., 140, 3844-3856, 2012.

Sun, C., Li, J., Jin, F., and Xie, F.: Contrasting meridional structures of stratospheric and tropospheric planetary wave variability in the Northern Hemisphere, Tellus, 66, 25303, doi:10.3402/tellusa.v66.25303, 2014.

Zadeh, L. A.: Fuzzy sets, Inform. Control., 8, 338-353, 1965. 